\title{
Femtosecond supercontinuum generation with noisy pumps in normal dispersion fibers with zero crossings
}

Rao Delanthabettu Shivarama, Shreesha; Genier, Etienne; Engelsholm, Rasmus Dybbro; Bravo Gonzalo, Ivan; Zhou, Binbin; Bowen, Patrick ; Moselund, Peter M. ; Sylvestre, Thibaut; Dudley, John M.; Bache, Morten

Total number of authors:

11

Published in:

Nonlinear Optics 2019

Link to article, DOI:

10.1364/NLO.2019.NM3A.2

Publication date:

2019

Document Version

Peer reviewed version

Link back to DTU Orbit

Citation (APA):

Rao Delanthabettu Shivarama, S., Genier, E., Engelsholm, R. D., Bravo Gonzalo, I., Zhou, B., Bowen, P., Moselund, P. M., Sylvestre, T., Dudley, J. M., Bache, M., \& Bang, O. (2019). Femtosecond supercontinuum generation with noisy pumps in normal dispersion fibers with zero crossings. In Nonlinear Optics 2019 [Paper NM3A.2] Optical Society of America (OSA). https://doi.org/10.1364/NLO.2019.NM3A.2

\section{General rights}

Copyright and moral rights for the publications made accessible in the public portal are retained by the authors and/or other copyright owners and it is a condition of accessing publications that users recognise and abide by the legal requirements associated with these rights.

- Users may download and print one copy of any publication from the public portal for the purpose of private study or research.

- You may not further distribute the material or use it for any profit-making activity or commercial gain

- You may freely distribute the URL identifying the publication in the public portal 


\title{
Femtosecond supercontinuum generation with noisy pumps in normal dispersion fibers with zero crossings
}

\author{
Shreesha Rao D. S. ${ }^{1}$, Etienne Genier ${ }^{2,3}$, Rasmus D. Engelsholm ${ }^{1}$, Iván B. Gonzalo ${ }^{1}$, Binbin Zhou ${ }^{1}$, Patrick \\ Bowen $^{2}$, Peter M. Moselund ${ }^{2}$, Thibaut Sylvestre ${ }^{3}$, John M. Dudley ${ }^{3}$, Morten Bache ${ }^{1}$, and Ole Bang ${ }^{1,2, *}$ \\ 1. DTU-Fotonik, Department of Photonics Engineering, Technical University of Denmark, Ørsteds Plads, 2800, Lyngby, Denmark. \\ 2. NKT Photonics A/S, Blokken 84, 3460 Birkerød, Denmark. \\ 3. Institut FEMTO-ST, UMR 6174 CNRS-Université de Franche-Comté, 25030 Besançon, France. \\ *oban@fotonik.dtu.dk
}

\begin{abstract}
We demonstrate surprising effects of technical pump laser fluctuations on the noise of a normal-dispersion fs-pumped supercontinuum and how the noise varies with power in fibers with a zero-dispersion at longer wavelengths. (c) 2019 The Author(s)
\end{abstract}

OCIS codes: (320.6629) Supercontinuum generation; (030.1640) Coherence.

\section{Introduction}

There has been a recent push toward understanding and lowering the noise of supercontinuum (SC) sources due to the increasing number of applications within spectroscopy, and imaging, such as in optical coherence tomography, photo-acoustic microscopy, and hyper-spectral imaging. One of the most promising approaches to lower the noise is to generate the SC in a fiber with weak all-normal dispersion (ANDi) using short fs pump pulses, in which case the spectral broadening can be dominated by the coherent processes of Self-Phase Modulation (SPM) and optical wavebreaking [1]. One requirements for generating a low noise ANDi SC like this is that the normal dispersion is weak in order to avoid parametric Raman noise [2]. Other key requirements to avoid Raman noise is that the pulse length and fiber length are sufficiently short [2]. Recent polarization studies demonstrated also that in addition the pump peak power needs to be low enough and the birefringence high enough to avoid detrimental noise from polarization mode instability (PMI) [3].

When these requirements are all satisfied an important question becomes: What is the influence of technical pump laser noise now that the SC generation process itself is coherent? Here we show that it strongly dominates quantum noise and becomes essential in determining the SC noise. In doing so we analytically and numerically explain the surprising experimental [4] and numerical [4,5] observation that the Relative Intensity Noise (RIN) of the SC at the pump wavelength, is actually lower than the RIN of the pump itself in this regime. Finally, if one want to use other pump wavelengths and thus other fibers than the traditional NKT Photonics ANDi fiber for 1064nm operation, does it lower the performance if the dispersion is not all-normal but becomes anomalous out at longer wavelengths? We answer this question by presenting the first single-material fiber suitable for ANDi SC generation at 1550nm and demonstrating experimentally and numerically the power levels one can use and the bandwidths one can achieve, before light crosses into the anomalous dispersion region and generates noisy solitons and Dispersive Waves (DWs).

\section{Results}

In Fig. 1(a) we show the dispersion of the standard ANDi fiber for $1064 \mathrm{~nm}$ pumping ( $1 \mu \mathrm{m}$ fiber) and our fiber for $1550 \mathrm{~nm}$ pumping $(1.5 \mu \mathrm{m}$ fiber), made with different rings of holes to reduce loss [4]. Given that we are in the regime of pulse and fiber parameters where the SC stays in a single polarization we use a scalar model in the form of a generalized nonlinear Schrödinger equation for our modelling [4]. We consider three sources of noise: (a) quantum noise $\delta_{Q}$, (b) laser amplitude fluctuations, $\delta_{A}$, and (c) laser pulse duration fluctuations that are anti-correlated with the amplitude as $\delta_{T}=-0.8 \delta_{A}$ or $\delta_{T}=-\delta_{A}$ (specific to the mode-locked laser). Here $\delta_{A}$ is a Gaussian distributions around zero giving fluctuation as a percentage of the amplitude. The peak power and pulse duration of a mode-locked laser are thus anti-correlated, which is extremely important for the SC noise, as we shall see. In the following we consider two cases: (1) the $1 \mu \mathrm{m}$ fiber pumped at $1054 \mathrm{~nm}$ with in-coupled peak power $\mathrm{P}_{0}=100 \mathrm{~kW}$, pulse length $\mathrm{T} 0=50 \mathrm{fs}$, and $\delta_{T}=$ $-0.8 \delta_{A}$ (found from datasheats for an Origami 10 from Onefive) [5], and (2) the $1.5 \mu \mathrm{m}$ fiber pumped at $1.55 \mu \mathrm{m}$ with a $90 \mathrm{MHz}$ (Toptica) laser with $\mathrm{T}_{0}=125 \mathrm{fs}$, a measured RIN of $1 \%$, and assuming $\delta_{T}=-\delta_{A}$ [4].

In Fig. 1(b) we consider the $1 \mu \mathrm{m}$ fiber for a standard amplitude noise of $\delta_{A}=0.5 \%$ and show the influence of the different types of noise in isolation and together. The periodic SPM spectral structure is evident in the RIN profile, but the important observations are: (1) the effect of quantum noise is negligible compared to that from the laser technical noise of $0.5 \%$, (2) The SC noise is lower when both amplitude and pulse duration fluctions are taken into account than 
when only one of them act in isolation, and (3) away from the spectral edges the RIN of the SC is lower than the $0.5 \%$ amplitude noise of the pump laser. Observation (3) is confirmed experimentally using the $1.5 \mu \mathrm{m}$ fiber, where the observed SC RIN of $0.61 \%$ at $1550 \mathrm{~nm}$ is lower than the measured $1 \%$ RIN of the $1550 \mathrm{~nm}$ pump laser. The important result (2), which explains (3), can be verified analytically [5], considering the exact solution for the envelope A(z,t) to the well-known model of SPM, and Fourier transforming (denoted by tilde) it to obtain the spectral intensity

$$
|\tilde{A}(\omega)|^{2}=\frac{\pi^{2}}{4} P_{0} T_{0}^{2} \operatorname{sech}^{2}\left(\pi T_{0} \frac{\omega-\omega_{0}+\Delta \omega}{2}\right)
$$

valid close to the outer SPM lope for the case when the frequency shift $\Delta \omega=-0.77 \gamma \mathrm{P}_{0} \mathrm{z} / \mathrm{T}_{0}$ is large, $\gamma$ being the fiber nonlinearity and $\omega_{0}$ the pump frequency [5]. At the peak, e.g., we therefore see that the amplitude and anti-correlated pulse duration will act oppositely and tend to cancel each other. The fact that the SC RIN is lower than the pump laser amplitude noise is thus to be expected from theory and due to the anti-correlated pulse duration noise.
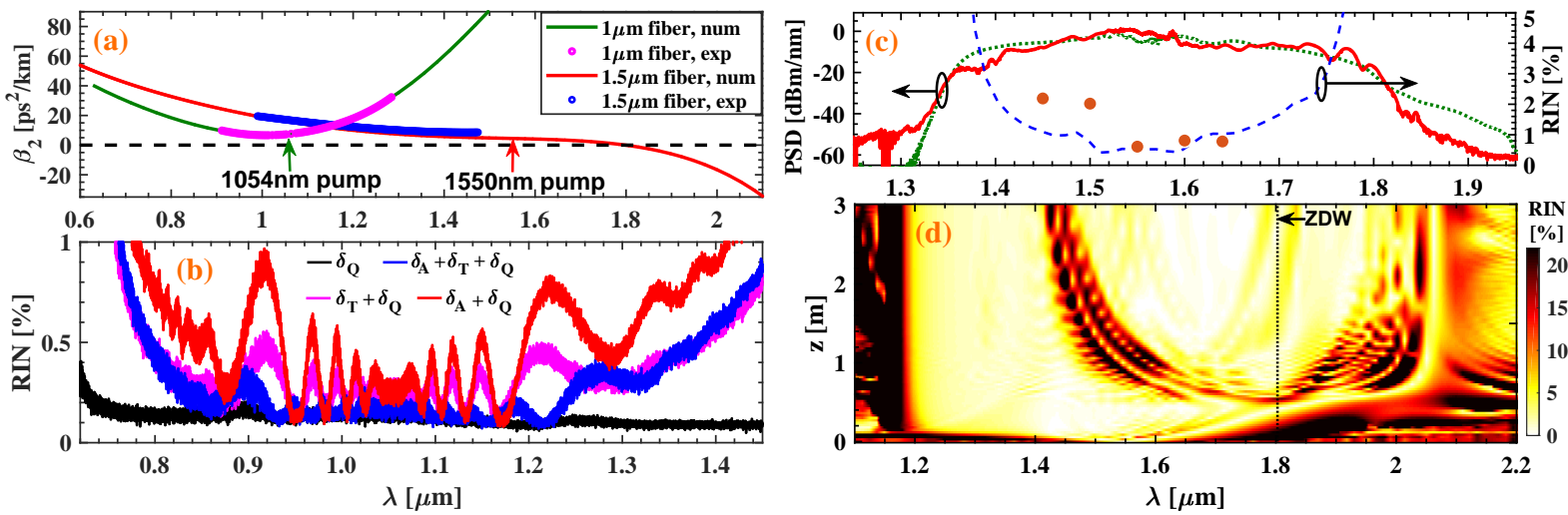

Fig. 1. (a) Numerically calculated (red+green) and experimentally measured (blue+magenta) dispersion. (b) Numerically calculated RIN out of $10 \mathrm{~cm} 1 \mu \mathrm{m}$ fiber for $\delta_{A}=0.5 \%$, taking into account $\delta_{Q}$ (black), $\delta_{Q}+\delta_{A}$ (red), $\delta_{Q}+\delta_{T}$ (magenta), and $\delta_{Q}+\delta_{A}+\delta_{T}$ (blue). (c) Experimentally measured SC out of $3 \mathrm{~m}$ of $1.5 \mu \mathrm{m}$ fiber with $\mathrm{P}_{0}=9 \mathrm{~kW}$ (full) and corresponding numerical simulation for $\delta_{A}=1 \%$ (dots) along with measured (red dots) and numerically found RIN (dashed). (d) Evolution of RIN along the length of $1.5 \mu \mathrm{m}$ fiber for $\mathrm{P}_{0}=45 \mathrm{~kW}$.

Let us turn to the $1.5 \mu \mathrm{m}$ fiber and the effect of the zero-dispersion wavelength (ZDW) it has at $1.8 \mu \mathrm{m}$ in order to get the weak flat dispersion around the pump seen in Fig. 1(a). For the maximum power of our laser, for which $\mathrm{P}_{0}=9 \mathrm{~kW}$, we observe in Fig. 1(c) that our modelling closely matches the measured spectrum and reasonably well the measured RIN values. The RIN of the laser was measured to be $1 \%$ and at this power level we see that power has not crossed the ZDW and that the SC RIN is low away from the edges, e.g., being below $2.2 \%$ for the measured values. The noise for long pulse SC is in comparison typically more than 50\%, so the ANDi SC is indeed low-noise. Given the accuracy of the modelling we used modelling to study higher powers and found that the power could be increased to about $\mathrm{P}_{0}=27 \mathrm{~kW}$ to provide a low noise $1550 \mathrm{~nm}$ pumped ANDi SC covering 1.24-1.8 $\mu \mathrm{m}$. In Fig. 1(d) we show the very interesting dynamics at a higher power of $\mathrm{P}_{0}=45 \mathrm{~kW}$ and see how the solitons generated above the $\mathrm{ZDW}$ when power crossed the ZDW, generates a localized high-noise DW, which moves through the otherwise low-noise ANDi SC below the ZDW. The presence of the ZDW is thus ok as long as the application does not require too high power.

Funding. European Union's Horizon 2020 research and innovation programme under the Marie Sklodowska-Curie grant agreement No 722380 (the project SUPUVIR), and grant agreement No 7326 (the project GALAHAD).

\section{References}

1. A. M. Heidt, A. Hartung, G. W. Bosman, P. Krok, E. G. Rohwer, H. Schwoerer, and H. Bartelt, "Coherent octave spanning near-infrared and visible supercontinuum generation in all-normal dispersion photonic crystal fibers," Opt. Express 19, 3775-3787 (2011).

2. A. M. Heidt, J. S. Feehan, J. H. V. Price, and T. Feurer, "Limits of coherent supercontinuum generation in normal dispersion fibers," J. Opt. Soc. Am. B 34, 764-775 (2017).

3. I. B. Gonzalo, R. D. Engelsholm, M. P. Sørensen, and O. Bang, "Polarization noise places severe constraints on coherence of all-normal dispersion femtosecond supercontinuum generation," Sci. Reports 8, 6579 (2018).

4. S. Rao D. S., R. D. Engelsholm, I. B. Gonzalo, B. Zhou, P. Bowen, P. M. Moselund, O. Bang, and M. Bache, "Ultra-low Noise Supercontinuum Generation with Flat Near-zero Normal Dispersion Fiber," arXiv preprint arXiv:1812.03877 (2018).

5. E. Genier, P. Bowen, T. Sylvestre, J. M. Dudley, P. M. Moselund, and O. Bang, "Amplitude noise and coherence degradation of femtosecond supercontinuum generation in all-normal-dispersion fibers," J. Opt. Soc. Am. B 36, A161-A167 (2019). 